\title{
Characterization, Optimization and Application of a Hyperspectral X-ray Camera
}

Frederic Van Assche ${ }^{1, *}$, Sander Vanheule ${ }^{1}$, Silvia Cipiccia ${ }^{3}$, Pieter Tack ${ }^{2}$, Ella De Pauw ${ }^{2}$, Laszlo Vincze $^{2}$, Luc Van Hoorebeke ${ }^{1}$ and Matthieu N. Boone ${ }^{1}$

1. UGCT-RP, Dept. Physics and Astronomy, Ghent University, Proeftuinstraat 86/N12, B-9000 Gent, Belgium

2. XMI, Dept. Chemistry, Ghent University, Krijgslaan 281/S12, B-9000 Gent, Belgium

3. Diamond Light Source

* Corresponding author, Frederic.VanAssche@UGent.be

There is an increasing research interest in combining X-ray spectroscopy with imaging. This is evident from the development of facilities combining both modalities in a single instrument [1,2]. One common way of exploiting spectral information in X-ray imaging is by modifying the source spectrum using techniques such as dual energy CT (DECT), monochromating optics, or using true mono-energetic sources. These options unfortunately often provide only limited spectral information or are infeasible for use in normal laboratory setups. Alternatively, detectors are being developed that combine spectroscopic capabilities with spatial resolving power [3-5]. The availability of these full-field energy resolving detectors is enabling the use of true spectroscopic X-ray imaging techniques at small-scale X-ray microscopy facilities, such as the UGent Centre for X-ray Tomography (UGCT).

At UGCT we operate the SLcam, a full-field hyperspectral camera prototype, jointly acquired with the Xray Micro-spectroscopy and Imaging group (XMI). This system uses a silicon CCD sensor with 264 by 264 pixels measuring $48^{2} \mu \mathrm{m}^{2}$. With its fully depleted $450 \mu \mathrm{m}$ silicon thickness a quantum efficiency of around $37 \%$ is obtained at $20 \mathrm{keV}$, with a developer specified energy resolution of $152 \mathrm{eV}$ or better at the manganese $\mathrm{K}_{\alpha}$ line [4]. The camera operates in photon counting mode where incident $\mathrm{X}$-rays generate deposited energy-dependent charge clusters spread over one or more pixels. These charges are amplified, digitized, and recombined into photon events with an associated energy and "centre-of-mass" location. This way a full energy spectrum is obtained for each pixel, making the system a true full-field hyperspectral camera.

Because the CCD sensor employed in the camera was originally developed for high sensitivity and low noise when used in low flux astronomical applications, and later adapted by PNSensor GmbH for use in spectroscopic X-ray fluorescence (XRF) and X-ray diffraction (XRD) measurements, significant challenges appear when faced with X-ray transmission imaging. Most prominently, the low acceptable photon fluxes impose very long integration times for transmission imaging, and the measured photon counts show a complex non-linear behaviour, both dependent on incident photon flux as well as not only single photon energy, but the full photon energy spectrum. [6]

In order to improve the performance of the SLcam, a complete new set of software was developed at UGCT. The machine housekeeping application was rewritten for improved hardware safety and stability, and a modern and modular acquisition and processing chain was developed based on small modular processes with clearly defined functions communicating using ZeroMQ. This enables the development of high performance distributed algorithms for online processing of frames at the full $400 \mathrm{~Hz}$ framerate. At any point from raw frames to finished images, intermediate results and diagnostic metrics can be extracted from the processing chain and stored for analysis. This opens up the full flexibility offered by the full- 
field spectral camera system and allows detector performance to be optimised even after acquisition.

To optimize the performance of this photon-counting detector, a set of parameters is determined to classify and analyse the clusters created by X-ray photons. These parameters not only need to include photon event information but also their relation to the properties of the detector and electronics, and should be usable for fast analysis of a great number of photons. While traditionally this camera has been calibrated and verified using the manganese $\mathrm{K}_{\alpha}$ and $\mathrm{K}_{\beta}$ lines from the decay of an Fe55 radioactive source, more data at different energies and fluxes was required. During a two-day beamtime investigating the spectral and spatial properties of the X-ray beam and optics at the I13-2 imaging beamline at Diamond Light Source (DLS) in Didcot, UK [7], datasets of raw frames were collected at different monochromator energies. Because the multi-layer monochromator (MLM) at I13-2 has its harmonics at non-exact multiples of the fundamental energy when using a Ruthenium strip, it becomes possible to split up double-counted fundamental photons from real photons with very similar energy in the harmonics, as shown in Figure 1. This provided us with photon cluster statistics both for actual photons as well as double counted photons at nearly identical energies, minimising the difference in most energy-dependent cluster properties and allowing investigation of double-counting mitigation techniques.

Through characterization of the photon response of the SLcam at a wide range of energies and fluxes, we aim to provide tunable parameters to tailor camera performance for each individual application and sample, even allowing multiple different operating modes to be used in parallel during a single acquisition. Finally, the effects of improved characterization and calibration of the system on current and future applications will be explored.

[1] B Laforce et al, Anal. Chem. 89 (2017) 10617

[2] G Martinez-Criado et al, J. Synchr. Radiat. 23(1) (2016) 344-352

[3] T Michel et al, Nucl. Instrum. Methods Phys. Res., Sect. A 589(2) (2009) 510-514

[4] O Scharf et al., Anal. Chem. 83 (2011), 2532-2538

[5] L Jones et al, Nucl. Instrum. Methods Phys. Res., Sect. A 604 (2009) 34-37

[6] M Boone, PhD thesis, Ghent University (2013)

[7] C. Rau et al., Phys Status Solidi 208(11) (2011), 2522-2525

The authors acknowledge funding from the Research Foundation - Flanders (FWO Research Project G0A0417N) and Diamond Light Source for time on beamline I13-2 under proposal MT19579.

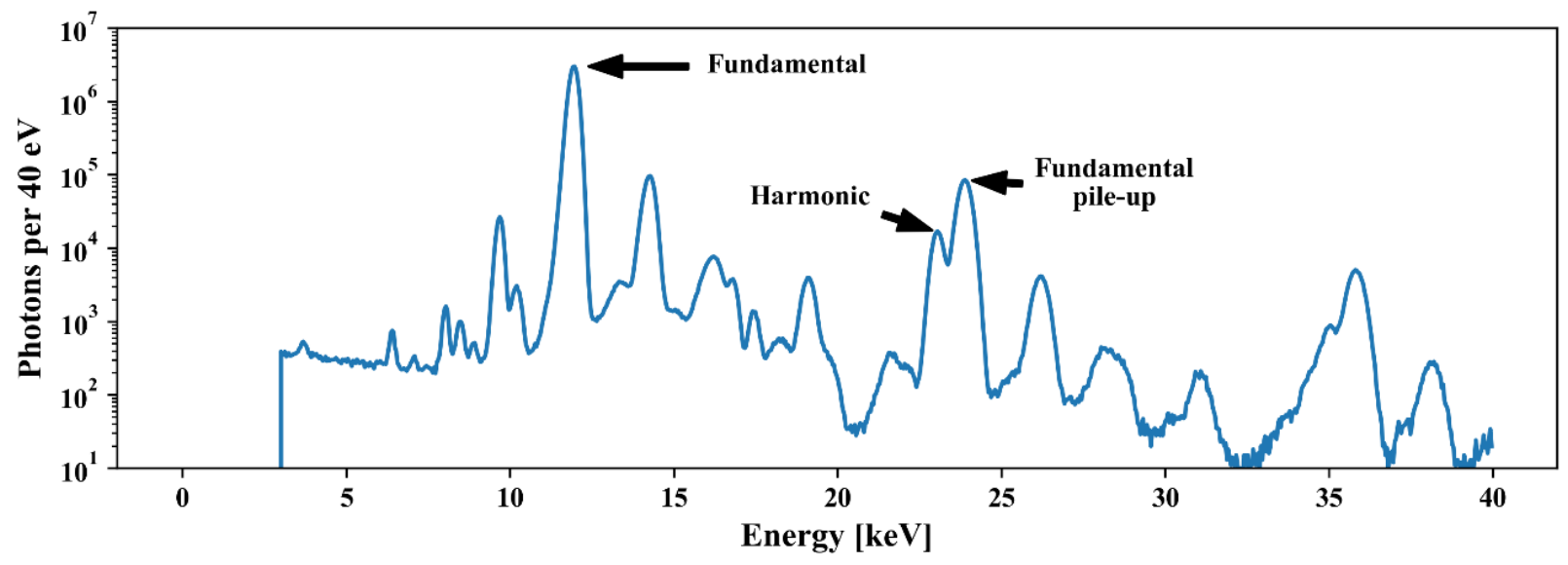

Figure. 1. Spectrum captured during beamtime at I13-2 showing split double counting and harmonic spectral peaks. 